\title{
HEPATIC AND RENAL HISTOPATHOLOGICAL AND ULTRASTRUCTURAL ALTERATIONS FOLLOWING EXPOSURE TO DIFFERENT GOLD NANOPARTICLE SIZES IN FEMALE PREGNANT RATS.
}

\author{
Othman F. Abdelzaher'; Ahmed Sabry S. Abdoon ${ }^{2}$; Mohamed I. Rady'i Mona M. Abd-Elgalil \\ El-Sayed $^{3}$; Ahmed B.M. Mehany ${ }^{1}$ and Fathy Elshaer Mohammed ${ }^{1}$ \\ ${ }^{1}$ Department of Zoology, School of Science, Al Azhar University, Nasr City 11884, Cairo, Egypt. \\ ${ }^{2}$ Department of Animal Reproduction, Veterinary Research Division, National Research Centre, \\ Dokki 12622, Cairo, Egypt. \\ ${ }^{3}$ Lecturer of Histology -Faculty of Medicine for Girls - Al-Azhar University.
}

\begin{abstract}
Nanoparticles (NPs) not only offer a great possibility for biomedical application, pharmaceutics, but also may be used for novel diagnostic and therapeutic approaches. Currently, there are no data available regarding to what extent the degree of toxicity of gold nanoparticles (AuNSs). This study aimed to investigate the histopathological and ultrastructural effect of AuNSs size on the liver and kidney of female pregnant rats. To achieve that gold nanoparticles of $10 \mathrm{~nm}, 25 \mathrm{~nm}$ and $50 \mathrm{~nm}$ were Interperitoneally injected in female rats at 48 hr during rat pregnancy. Forty adult female albino rats were divided into four equal groups: The first one served as a control group, the second received $75 \mathrm{mg} / \mathrm{kg} /$ day AuNSs as $10 \mathrm{~nm}$ in size, the third received 75 $\mathrm{mg} / \mathrm{kg} /$ day AuNSs as $25 \mathrm{~nm}$ in size and the fourth received $75 \mathrm{mg} / \mathrm{kg} /$ day AuNSs as $50 \mathrm{~nm}$ in size, All groups were scarified after 19days from gestation where liver and kidney specimens were collected at the end of the experiment for histological and ultrastructural examination, AuNSs treated groups revealed wide spread histological and ultrastructural alterations in the liver and kidney structure as dilated and congested hepatic blood vessels, widespread vacuolated and ballooned hepatocytes with early apoptotic changes, pyknotic hyper chromatic irregular nuclei with deteriorated chromatin condensation. The distal convoluted tubule had shrunken pyknotic nuclei and margination of heterochromatin with irregular nuclear envelope. This study conculcated that all size of AuNSs caused side effect in the liver and kidney of pregnant rates.
\end{abstract}

Key words: Gold nanoparticles, pregnant rats, liver, kidney.

\section{INTRODUCTION}

Nanoparticles have a wide range of applications due to their unique properties that it can easily enter the cells and caused some toxicity (Connor et al., 2005). The AuNSs imply that they could get close to a biological target of interest. The environmental impact of NPs is evident; however, their nanotoxicity due to the reduction in size to nanoscale is rarely discussed. AuNSs may serve as a promising model to address this size-dependent toxicity, since gold is extraordinarily biocompatible. There are differing reports of the extent of the toxic nature of these particles owing to the different modifications of AuNSs, surface functional attachments, shape and diameter size of the Nano spheres (Takahashi et al., 2006 and Pan et al., 2007). All gold nanoparticle sizes $(10-250 \mathrm{~nm})$ were demonstrated to be present in blood, liver and spleen after $24 \mathrm{~h}$ from injection, a clear difference was observed between the distribution of the $10 \mathrm{~nm}$ particles and the larger particles. The $10 \mathrm{~nm}$ particles were present in various organ systems including blood, liver, spleen, kidney, testis, thymus, heart, lung and brain, whereas the larger particles were only detected in the blood, liver and spleen. The distribution of gold nanoparticles in tissue is size-dependent (De Jong et al., 2008). The most frequent reason for toxicity of AuNSs is accumulation in extracellular spaces and its solubility in intracellular spaces. AuNSs activate a kind of released oxygen in the intercellular space and reduces the function of living cells. These released oxygen from AuNSs action, prevent the proper functioning and growth of living cells. (Studer et al., 2010 and Gunawan et al., 2011). The AuNSs were found to induce a significant oxidative stress in liver of rats, and 
it plays important roles in inflammatory, genotoxic and proliferative responses (Jia $\boldsymbol{e t}$ al., 2009 and Tedesco et al., 2010). The AuNSs size and exposure duration effect on the liver and kidney function of rats: in vivo have not been well documented before. In the present study we aimed to address the particlesize effect on the liver and kidney function in an attempt to cover and understand the toxicity tool of AuNSs.

\section{MATERIALS AND METHODS:}

\section{Gold nanoparticles}

Gold nanoparticles (10, 25 and $50 \mathrm{~nm}$ particle size) were obtained from (National Research Center, Cairo, Egypt).

\section{Synthesis and characterization of gold nanospheres of 10, 25 and $50 \mathrm{~nm}$}

The Au nanosphere shapes were produced by ascorbic acid mediated reduction method. For synthesis of AuNSs with a diameter of about $25 \mathrm{~nm}$ (AuNSs 25), growth solutions of AuNSs25 (7.2 mL of $0.1 \mathrm{M} \quad \mathrm{CTAB}$ and $0.225 \mathrm{~mL}$ of $0.01 \mathrm{M} \mathrm{HAuCl}_{4} \cdot 3 \mathrm{H}_{2} \mathrm{O}$ ) and AuNSs $50(6.4 \mathrm{~mL}$ of $0.1 \mathrm{M} \mathrm{CTAB}$ and $0.200 \mathrm{~mL}$ of $\left.0.01 \mathrm{M} \mathrm{HAuCl}_{4} \cdot 3 \mathrm{H}_{2} \mathrm{O}\right)$ were separately mixed with solution of fresh ascorbic acid $(0.050 \mathrm{~mL}$, $0.1 \mathrm{M})$. After that, $0.1 \mathrm{~mL}$ and $0.026 \mathrm{~mL}$ of seed solution were transferred to the stirring mixtures, respectively. Finally, these solutions were stirred strongly for $10 \mathrm{sec}$ until a wine-red solution was obtained. The mixtures were left undisturbed overnight. According to (Turkevich et al 1951)

\section{Animals}

40 adult female albino rats (12 weeks old, $200 \pm 20 \mathrm{gm}$ ) were purchased from the Egyptian Holding Company for Biological Products and Vaccines (VACSERA). Animals were housed individually in clean plastic cages with steel toppings (1:1 male female ratio) with $12 \mathrm{hrs}$ day and night cycle in the animal house, the temperature ranged between $20-25^{\circ} \mathrm{C}$ with a relative humidity of $55 \pm 5 \%$. Food and water were added ad libitum. After one week of adaptation, female rats were mated overnight (each male rat was mated with 3 females). Day 1 of pregnancy was defined as the day in which the mucous plug was present and spermatozoa were found in vaginal smears.

Experimental design: Animals were randomly divided into four groups (10 rats in each group) as followous: First serve as control group in which pregnant rats were injected with normal saline solution intraperitoneally (i,p,) $48 \mathrm{hr}$ after mating. Group 1 (AuNSs injected groups), in which pregnant rats were i.p. injected with $75 \mu \mathrm{g} / \mathrm{kg}$ weight of $10 \mathrm{~nm}$ AuNSs. Group 2(AuNSs injected groups), in which pregnant rats were i.p. injected with75 $\mu \mathrm{g} / \mathrm{kg}$ weight of $25 \mathrm{~nm}$ AuNSs. Group 3(AuNSs injected groups), in which pregnant rats were i.p. injected with75 $\mu \mathrm{g} / \mathrm{kg}$ weight of $50 \mathrm{~nm}$ AuNSs. $48 \mathrm{hr}$ after mating, All groups were scarified on Day-19 of pregnancy.

\section{Histological studies:}

Tissues of liver and kidney of the treated and control rats were eviscerated, fixed in $10 \%$ formalin solution, dehydrated in ascending grades of ethyl alcohol, cleared in xylol and embedded in paraffin wax. Several sections of 5 microns thickness were prepared, Mounting and staining were carried out using Haematoxylin and Eosin for routine histological examination. The sections were examined using a research microscope supplied with an eyepiece micrometer according to (Davenport, 1960).

\section{Electron microscopy:}

The fresh hepatic and kidney tissues were primarily fixed by immersion in 5\% glutaraldehyde and then secondarily fixed by immersion in $1 \%$ osmium tetroxide, then washed in $0.1 \mathrm{M}$ cacodylate buffer. Fixed and washed specimens were dehydrated through an ascending grade of alcohol. The absolute alcohol was replaced by propylene oxide. Dehydrated specimens were infiltrated with the resin mixture through a graded series in glass vials with polypropylene caps, and then transferred to an oven. The mounted blocks 
were trimmed with razor blades using Ultra microtome. Semi thin sections $(1 \mu \mathrm{m}$ thick) were cut using glass knives and stained with toluidine blue. Ultra-thin sections $(50-80 \mathrm{~nm}$ thick) were cut using glass knives and the sections floated on water surface picked up on copper grids. The sections were double stained in uranyl acetate followed by lead citrate. Stained sections on grids were washed with $0.02 \mathrm{~N} \mathrm{NaOH}$ followed by distilled water and left on a filter paper in a petri dish (Johannessen, 1978) prior to examination with a JEOL 1010 Transmission Electron Microscope at the Regional Center for Mycology and Biotechnology (RCMB), AlAzhar University.

\section{RESULTS:}

Liver: Microscopic examination of liver sections from the control group revealed normal hepatic lobulations. The hepatocytes were arranged in anastomosing cords, radiating from the central vein to the periphery of the classical hepatic lobule, separated from each other by narrow elongated blood sinusoids in which the von Kupffer cells could be detected. The hepatocytes appeared polyhedral in shape with an eosinophilic granular cytoplasm and rounded central, vesicular nuclei with prominent nucleoli. Some hepatocytes were binucleated. At the corners of the hepatic lobules, the portal tract appeared to contain a branch of the hepatic artery, portal vein, lymph vessels and bile duct. The hepatic bile duct was lined with cubical epithelial cells (plate 1; A, B). Liver treated with $10 \mathrm{~nm}$ AuNSs showed dilated and congested hepatic blood vessels, vacuolated and ballooned hepatocytes with early apoptotic nuclei. There was extensive mononuclear cellular infiltration surrounding the bile ductules, and compensatory proliferated bile ducts could be detected at portal tract area. Kupffer cells were hypertrophied within the dilated blood sinusoids in some areas (plate 1; C, D). Also pregnant rates treated with $25 \mathrm{~nm}$ AuNSs revealed widespread preserved the normal hepatic architecture. However, degenerative and apoptotic changes were encountered in a few hepatocytes. Mononuclear cellular infiltration and bile duct proliferation were also encountered in the periportal area (plate 2; A, B). Furthermore the examination of liver for pregnant rats treated with $50 \mathrm{~nm}$ AuNSs, designated dilated, mild congested hepatic blood vessels surrounded with mononuclear cellular infiltration. Most hepatocytes preserved the normal hepatic architecture. However, hydropic degeneration and apoptotic changes were encountered in a few hepatocytes. The sinusoids were dilated with lymphocytic infiltration and hypertrophied Kupffer cells were encountered in some of them (plate 2; C, D).

\section{Transmission electron microscopic results:}

For electron transmission microscopic examination, samples of the liver from the control group revealed a normal ultrastructure of hepatocytes, enclosing central rounded euochromatic nuclei with well-defined nucleoli. The cytoplasm of the hepatocytes contained well-developed flattened cisternae of rough endoplasmic reticulum studded with ribosomes, numerous rounds, ovoid intact mitochondria and scattered glycogen granules. Bile canaliculi between adjacent hepatocytes contained microvilli and a junctional complex connecting these adjacent hepatocytes was observed, contained microvilli and von Kupffer cells, could be easily detected (plate 3; A, B and C). The ultrastructure of hepatic tissues from pregnant rats treated with $10 \mathrm{~nm}$ AuNSs showed marked cytopathological changes where hepatocytes, displayed euochromatic nucleus with margination of heterochromatin. Others displayed dramatic pyknotic hyperchromatic irregular nucleus with deteriorated chromatin condensation, the cytoplasm was highly vacuolated with rarified appearance and nearly devoid of organelles. Smaller stacks of 
fragmented rough endoplasmic reticulum and abnormal mitochondria that appeared swollen with complete loss of internal matrices and disrupted cristae could be detected. Further, Disse's spaces contained Von Kupffer cell, between the vacuolated hepatocytes showing numerous vacuoles and a large number of electron dense lysosomes (plate 3; D, E and F). However the ultrastructure of liver section from $25 \mathrm{~nm}$ AuNSs group showed almost normal ultrastructural appearance nearly similar to control group apart from few cytoplasmic vacuoles, electron lucent lipid droplets and many membranous electron dense lysosomes. Bile canaliculi between adjacent hepatocytes with absent microvilli could be seen. The space of Disse, containing microvilli and von Kupffer cell containing large number of lysosomes, could be easily detected (plate 4; A, B and C). The ultrastructure of liver from pregnant rats treated with 50nm AuNSs showed almost normal ultrastructural appearance nearly similar to those of $25 \mathrm{~nm}$ AuNSs group apart from more cytoplasmic vacuoles, larger electron lucent lipid droplets and more membranous electron dense lysosomes. Bile canaliculi between adjacent hepatocytes with absent microvilli could be seen. The space of Disse, containing microvilli and von Kupffer cell containing large number of lysosomes, could also be detected (plate 4; D, E and F).

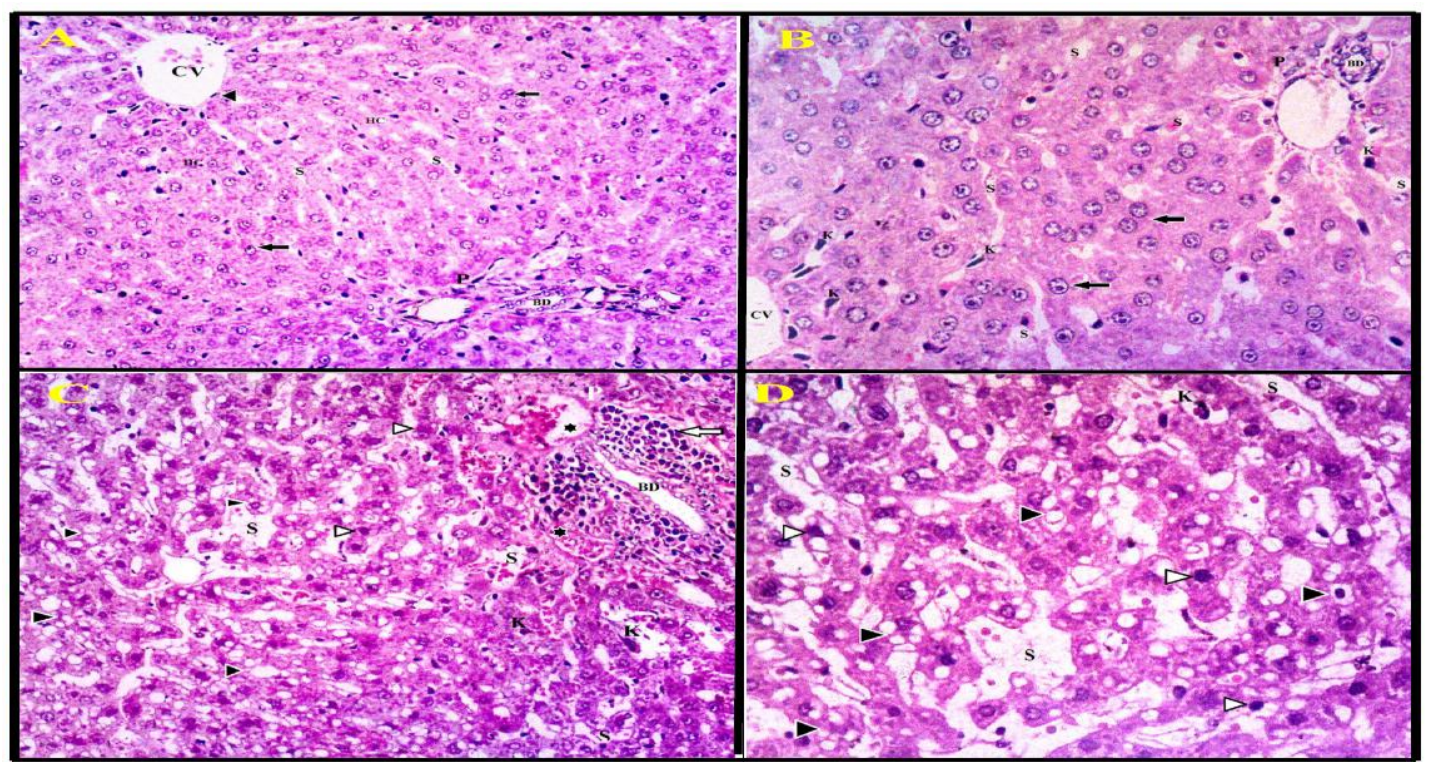

Plate 1 : ( A, B): photomicrograph of a liver section of the control group showing normal hepatic cords (HC) arrangement around the central vein (CV), separated by blood sinusoids (S). Notice, flattened endothelial lining of the central vein (arrow head 4), polyhedral eosinophilic granular hepatocytes with central rounded vesicular one or two nuclei (black arrow $\boldsymbol{\omega})$. The portal triad $(\mathrm{P})$ at the corner of hepatic lobule showing a branch of hepatic artery, portal vein and bile duct (BD) appeared lined with cubical epithelial cells, (B): central vein (CV), polyhedral eosinophilic hepatocytes with rounded vesicular one or two nuclei (black arrow $\boldsymbol{-})$, Von Kupffer cells (K) within the blood sinusoids (S). The portal triad (P) at the corner of hepatic lobule had a branch of hepatic artery, portal vein and bile duct (BD) lined with low cubical epithelial cells is also observed (H\&E X 200, 400). (C, D): photomicrograph of a liver section of pregnant female albino rat of $10 \mathrm{~nm}$ AuNSs-treated rat displaying widespread vacuolated and ballooned hepatocytes (black arrow head - ). Hepatocytes with apoptotic changes had dark eosinophilic cytoplasm and small deeply stained pyknotic nuclei (white arrow head $\triangleright$ ). Dilated and congested blood vessels (star *) with extensive mononuclear cellular infiltration ( $\diamond \quad$ ) and proliferated bile ductule (BD) are observed at the portal tract area (P). Notice hypertrophied Kupffer cells (K) within the dilated blood sinusoids (S), (D): vacuolated and ballooned hepatocytes (black arrow head 4 ). Hepatocytes with apoptotic changes had dark eosinophilic cytoplasm and small deeply stained nuclei (white arrow head $\triangleright$ ). Notice hypertrophied Kupffer cells (K) within the dilated blood sinusoids (S) (H\&E X 200, 400). 


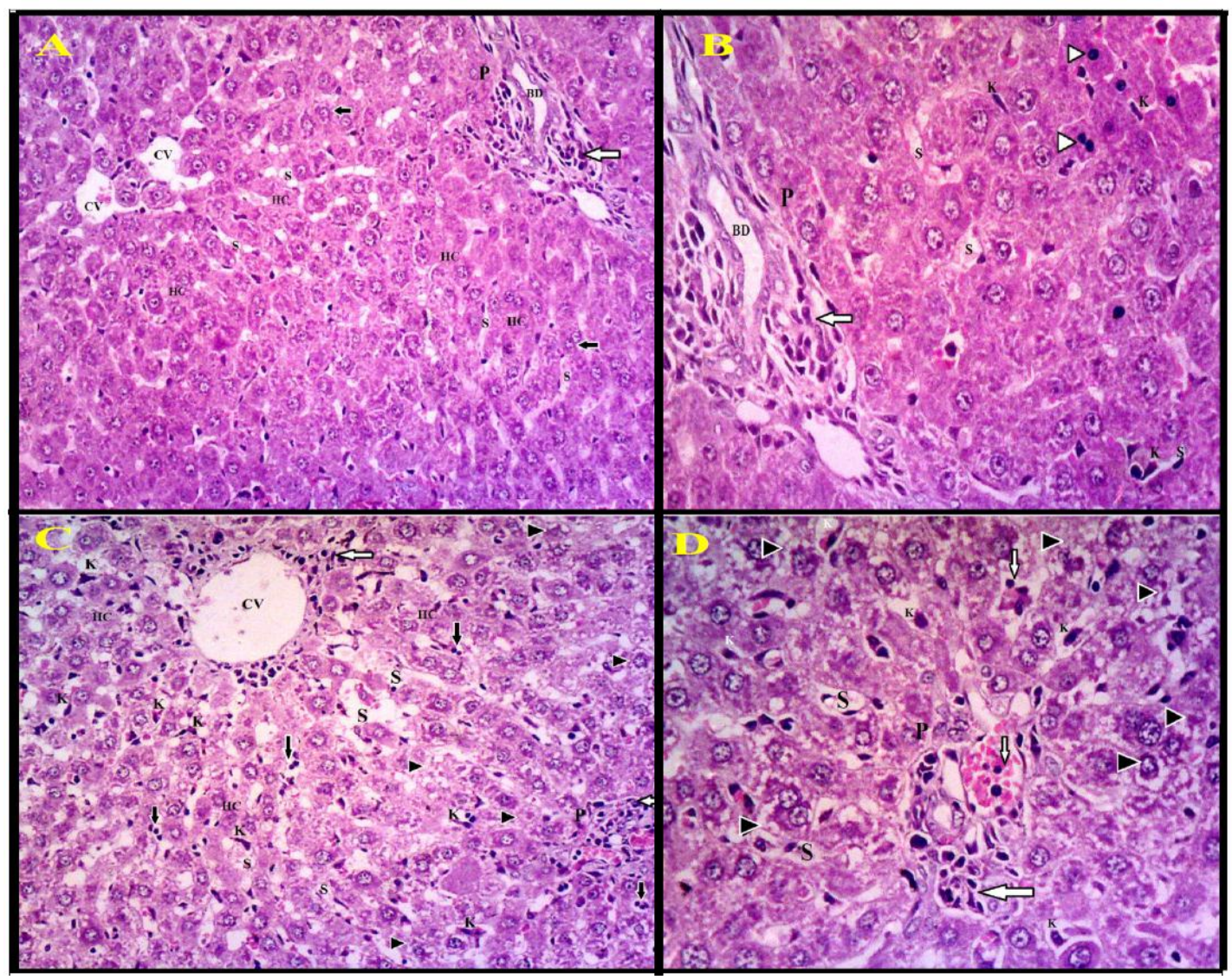

Plate 2:( A , B) A: photomicrograph of a liver section of $25 \mathrm{~nm}$ AuNSs-treated rat showing preservation of hepatic architecture, hepatic cords (HC) arrangement around the central vein (CV) and separated by blood sinusoids (S), polyhedral eosinophilic hepatocytes with central rounded vesicular one or two nuclei (black arrow $\bullet$ ) Notice, mononuclear cellular infiltration (white arrow $\diamond$ ) with dilated bile duct (BD) in the portal tract area (p), (B): mononuclear cellular infiltration (white arrow $\diamond)$ with dilated bile duct (BD) in the portal tract area (p). Few hepatocytes showed apoptotic degeneration with deeply eosinophilic cytoplasm and small deeply stained pyknotic nuclei (white arrow head $\triangleright$ ). Notice Kupffer cells (K) within the blood sinusoids (S) (H\&E X 200, 400). (C, D) C: photomicrograph of a liver section of pregnant female albino rat of $50 \mathrm{~nm}$ AuNSs-treated rat showing preservation of hepatic architecture. Hepatic cords (HC) were separated by dilated blood sinusoids (S). Notice, few vacuolated and ballooned hepatocytes (black arrow head mononuclear cellular infiltration (white arrow $\diamond)$ was observed around the dilated central vein $(\mathrm{CV})$ and at the portal tract area $(\mathrm{p})$ that contained congested dilated portal vessels, lymphocytic infiltration $(\sqrt{ })$ and hypertrophied Kupffer cells (K) within the dilated blood sinusoids (S) are also observed, (D): showing few vacuolated and ballooned hepatocytes (black arrow head), mononuclear cellular infiltration (white arrow $\diamond$ ) at the portal tract area (p) containing congested dilated portal vessels. Lymphocytic infilteration ( $\checkmark$ ) and hypertrophied Kupffer cells (K) within the dilated blood sinusoids (S) are also observed (H\&E X 200, 400). 


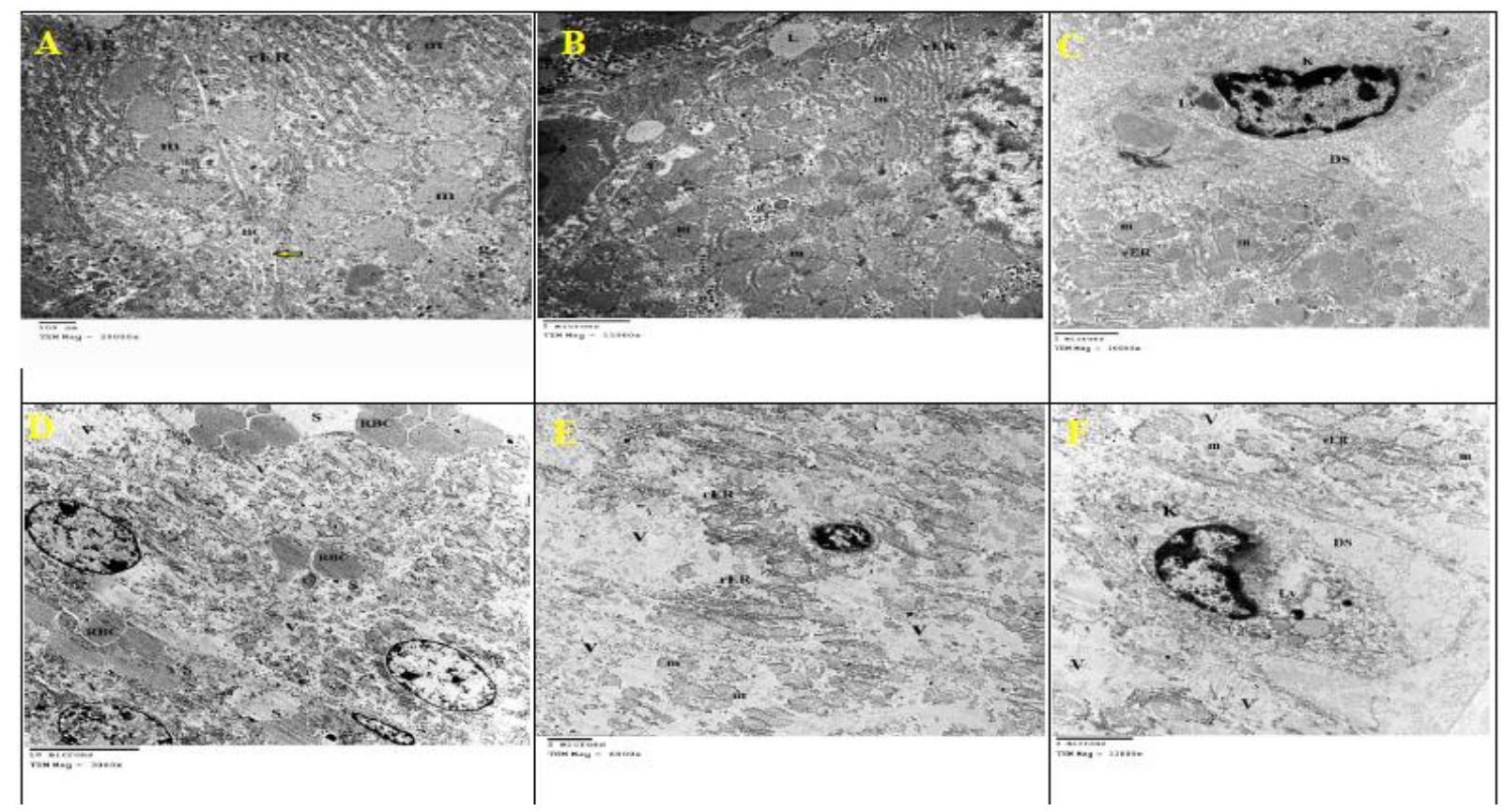

Plate 3: (A, B and C): Electron micrograph of a hepatic cell from the control group showing bile canaliculi (BC) between adjacent hepatocytes containing microvilli (yellow arrow). Notice a part of the adjacent hepatocytes cytoplasm contained mitochondria $(\mathrm{m})$ of variable shapes and sizes, rough endoplasmic reticulum (rER), and glycogen granules (g). (B): Hepatocytes with euchromatic nucleus. Notice, the parallel tubules of rough endoplasmic reticulum (rER) between many mitochondria $(\mathrm{m})$ of variable shapes and sizes, glycogen granules $(\mathrm{g})$, and electron-lucent lipid droplets (L). Notice the microvilli (mv) in the space of Disse. (C): Kupffer cell (K) in the space of Disse (DS) contains many electron-dense lysosomes (Ly). Notice, mitochondria (m) of variable shapes and sizes and the rough endoplasmic reticulum (rER) in a cytoplasm of the adjacent hepatocyte (Magnification x20000, 12000 and 10000).(D, E and F): Transmission electron micrograph of the liver section of $10 \mathrm{~nm}$ AuNSs-treated rat displaying highly vacuolated hepatocytes with blood sinusoids in between (S) containing red blood corpuscles (RBCs). The nuclei $(\mathrm{N})$ are euochromatic and the rarified cytoplasm is highly vacuolated (V) containing few degenerated organelles, highly vacuolated hepatocytes (V) with rarified cytoplasm. (E): Showing small sized pyknotic hyperchromatic nucleus with chromatin clumps $(\mathrm{N})$. Notice, stacks of fragmented rough endoplasmic reticulum and abnormal swollen mitochondria in the rarified cytoplasm, (F): Showing highly vacuolated kupffer cell (K) containing many electron-dense lysosomes (Ly) in the space of Disse (DS) surrounded by vacuolated hepatocytes (V). Notice, fragmented rough endoplasmic reticulum (rER) and abnormal swollen mitochondria $(\mathrm{m})$ in the rarified cytoplasm (Magnification X3000, 6000 and 12000). 


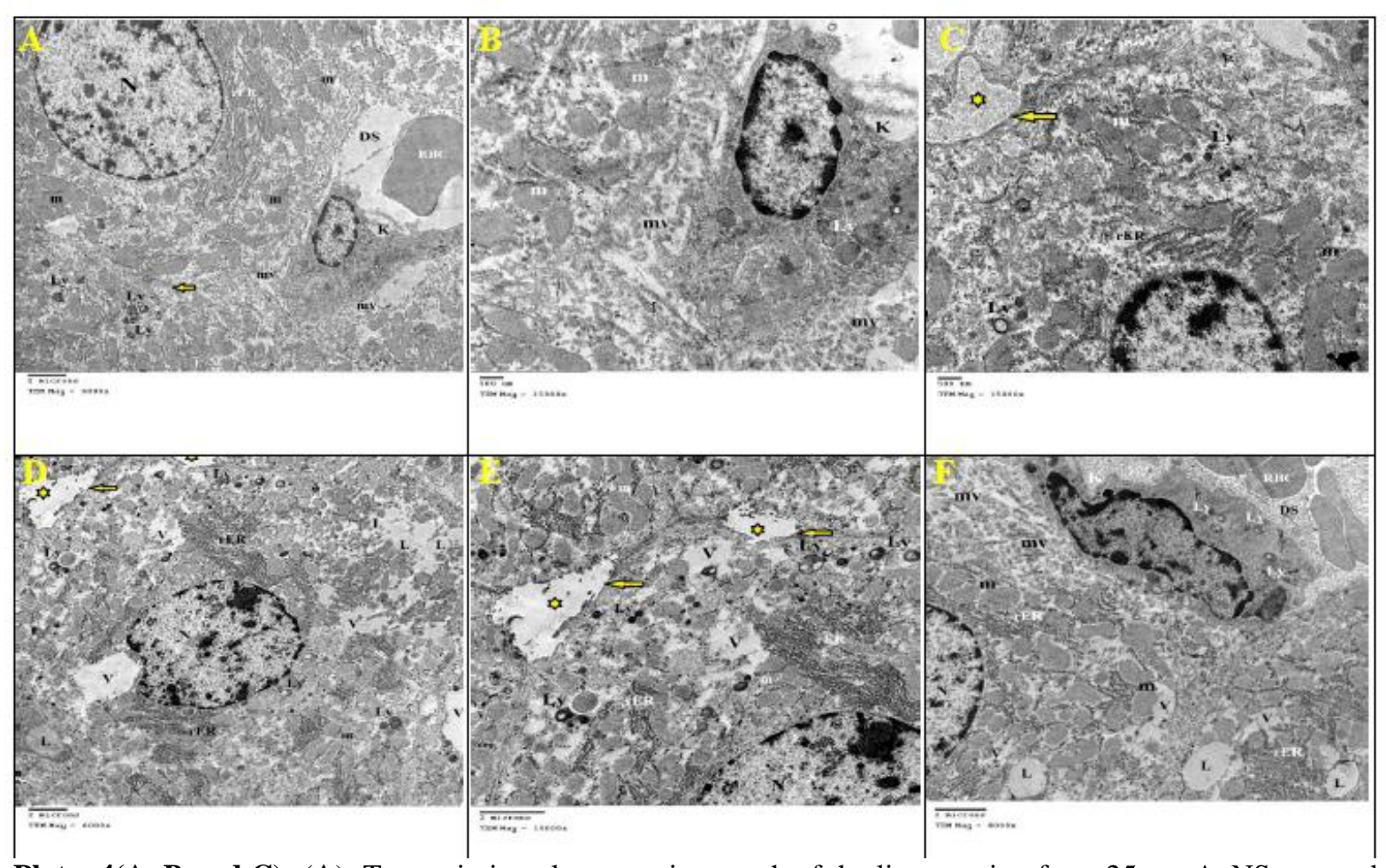

Plate: 4(A, B and C): (A): Transmission electron micrograph of the liver section from $25 \mathrm{~nm}$ AuNSs-treated pregnant rat displaying hepatocyte with euchromatic nucleus $(\mathrm{N})$, rough endoplasmic reticulum (rER) around the nucleus, between many mitochondria $(\mathrm{m})$ of variable shapes and sizes and many electron-dense lysosomes (Ly). Notice bile canaliculi $(\smile$ ) between adjacent hepatocytes. Space of Disse (DS) containing microvilli, Kupffer cell $(\mathrm{K})$ and red blood corpuscle (RBC), (B) :displaying a Kupffer cell (K) in the space of Disse (DS) containing many electron-dense bodies and lysosomes (Ly). Notice the microvilli (mv) in the space of Disse. Mitochondria $(\mathrm{m})$ of variable shapes and sizes are also evident in adjacent hepatocyte, displaying bile canaliculi $(\sqrt{ })$ between adjacent hepatocytes. (C): Notice the absence of microvilli in the lumen of the bile canaliculi ( $\sqrt{\mathbf{3}}$ ), parallel tubules of rough endoplasmic reticulum (rER) between mitochondria (m) of variable shapes and sizes and many electron-dense lysosomes (Ly) in hepatocyte cytoplasm (Magnification x6000, 15000 and 15000). (D, E and F): electron micrograph of the liver section from $50 \mathrm{~nm}$ AuNSs-treated pregnant rat displaying hepatocyte with euchromatic nucleus $(\mathrm{N}) .(\mathbf{E})$ : Notice many vacuoles $(\mathrm{V})$ variables in size and shape, electron lucent lipid droplets (L), rough endoplasmic reticulum (rER) around the nucleus and between many mitochondria $(\mathrm{m})$ of variable shapes and sizes and numerous electron-dense lysosomes (Ly). Notice, dilated bile canaliculi ( $\checkmark$ ) between adjacent hepatocytes with the absence of microvilli in the lumen of the bile canaliculi ( $\widehat{\mathbf{z}}$ ), euchromatic nucleus. Vacuoles $(\mathrm{V})$, rough endoplasmic reticulum (rER), many mitochondria $(\mathrm{m})$ of variable shapes and sizes and many electron-dense lysosomes (Ly). (F): Notice, dilated bile canaliculi $(<$ ) between adjacent hepatocytes with the absence of microvilli in the lumen of the bile canaliculi ( $\sqrt{\mathbf{3}}$ ), displaying a Kupffer cell (K) containing many electron-dense lysosomes (Ly) in the space of Disse (DS) . Notice the microvilli (mv) and red blood corpuscles (RBC) in the space of Disse. The hepatocyte displaying vacuoles $(\mathrm{V})$, a part of euchromatic nucleus $(\mathrm{N})$, rough endoplasmic reticulum (rER), many mitochondria $(\mathrm{m})$ of variable shapes and sizes and lipid droplets $(\mathrm{L})$, with rough endoplasmic reticulum (rER) (Magnification x6000, 10000 and 8000).

Kidney: Section from control group showed a normal appearance of the Malpighian renal corpuscles with normal glomerular structure composed of capillary tufts surrounded by visceral and parietal layers of Bowman's capsule which were separated by Bowman's spaces. Proximal convoluted tubules with a characteristic narrow lumen were lined by a few cuboidal epithelial cells, while, wider distal convoluted tubules were lined by more cubical cells, Peritubular capillaries appeared separating convoluted tubules. Sections in the 
renal medulla revealed wider collecting tubules with a thin wall lined by low cubical cells (plate 5: A, B). Pregnant rat treated with $10 \mathrm{~nm}$ AuNSs demonstrated glomerular, tubular and interstitial histological alterations, congested renal and peritubular capillaries was observed together with Shrunken renal corpuscles with collapsed tuft and wide Bowman's space. Some glomeruli appeared lobulated. Tubulo interstitial Hydropic degeneration was present, in the form of tubular dilatation, cloudy swelling and vacuolated cytoplasm of the lining cells. This alteration was more prominent in the proximal convoluted tubules than the distal ones. Some of the tubules showed apoptotic changes, in which tubular nuclei showed shrinkage, pyknosis with chromatin condensation and even loss of nuclei in some cells. Sloughing of the tubular cells with appearance of hyaline casts inside some tubules was observed and some collecting tubules were lined with flat dark nuclei (plate 5: C, D, H and E). Occasional glomerular congestion was seen in the kidney sections from rats exposed to $25 \mathrm{~nm}$ AuNSs. The congested glomeruli showed capillary dilatation. Renal tubules appeared dilated and showed degenerative changes mostly cloudy swelling, vacuolated cytoplasm and pyknotic nuclei of the lining tubular epithelium. Sloughing of the tubular cells inside their lumen and inside some dilated collecting tubules were also observed that appeared lined with flat dark nuclei. Moreover, congestion of peritubular capillaries and interstitial mononuclear cellular infiltration were also noticed in some areas (plate 6: A, B and $\mathrm{C}$ ). Also examined sections from $50 \mathrm{~nm}$ AuNSs, revealed partial preservation in the normal histological structure of the renal glomeruli and tubules. However, some renal histolpathological lesions appeared more or less similar to those of $25 \mathrm{~nm}$ AuNSs group. Congested glomeruli with capillary dilatation and occasional empty spaces lined by squamous cells were noticed. Some renal tubules were mildly dilated, showed degenerative changes mostly cloudy swelling and others showed pyknotic nuclei. (plate6: D and E).

Ultrastructural investigations of renal tissue from control group showed a normal ultrastructure of renal corpuscle, tubular epithelial cell layer and basement membrane of the proximal convoluted tubule. The nuclei were rounded euchromatic. Its cytoplasm contained intact cell organelles, including numerous normal elongated mitochondria, basal membrane infoldings and a few lysosomes. The luminal surface of the cells had long densely packed microvilli. The kidney glomerular tissue showed multiple glomerular capillaries, podocytes with central nucleus and characteristic well-formed primary and secondary foot processes with regular spacing between them resting on the glomerular basement membrane. Normal mesangial cells, with electron-dense cytoplasm surrounded by its mesangial matrix supporting the wall of the capillary were observed (Plate 7: A, B and C). all examined ultrathin sections from $10 \mathrm{~nm}$ AuNSs, treated group revealed the renal epithelium had a thickened basement membrane with decreased apical microvilli, pale rounded nucleus with margination of heterochromatin and the cytoplasm was nearly devoid of organelles except from many pinocytotic vesicles, membranous electron dense secondary lysosomes and swollen mitochondria with destroyed cristae and loss of internal matrices. The distal convoluted tubule had shrunken pyknotic nuclei and margination of heterochromatin with irregular nuclear envelope. The tubular cytoplasm revealed hydropic degeneration, vacuolation. Basal infoldings are apparent in some areas. The kidney glomerular tissue displayed multiple glomerular capillaries, swelling of primary foot processes and fusion of the secondary foot processes of the podocyte in certain areas with irregular spacing between them and apparent thickening of basement membra were detected (Plate7: D, E and F). Ultrastructural cytopathological changes in renal sections from $25 \mathrm{~nm}$ AuNSs treated group, evidenced long densely packed microvilli at the luminal 
surface of the proximal tubular cells. Mitochondria appeared normal with intact cristae and electron dense internal matrices. In addition, congested glomerular capillaries, swollen primary foot processes of the podocytes and fusion of secondary foot processes in certain areas appeared with irregular spacing between them and apparent thickening of basement membrane (plate 8: A, $\mathrm{B}$ and $\mathrm{C})$.

Ultrastructural cytopathological changes in renal sections from50nm AuNSs treated group were nearly similar to those of $25 \mathrm{~nm}$ AuNSs treated group. The proximal tubular cells had a thickened basement membrane with decreased disrupted apical microvilli and the cytoplasm

contained pinocytotic vesicles, membranous electron dense lysosomes, swollen mitochondria with intact cristae and electron dense internal matrices and rounded nucleus with margination of heterochromatin. The distal convoluted tubule had shrunken pyknotic nuclei and margination of heterochromatin with irregular nuclear envelope. Basal infoldings are apparent in some areas. The kidney glomerular tissue displayed multiple congested glomerular capillaries, swelling of primary foot processes and fusion of the secondary foot processes of the podocyte in certain areas with irregular spacing between them and apparent thickening of basement membrane (Plate 8: D, E and F).

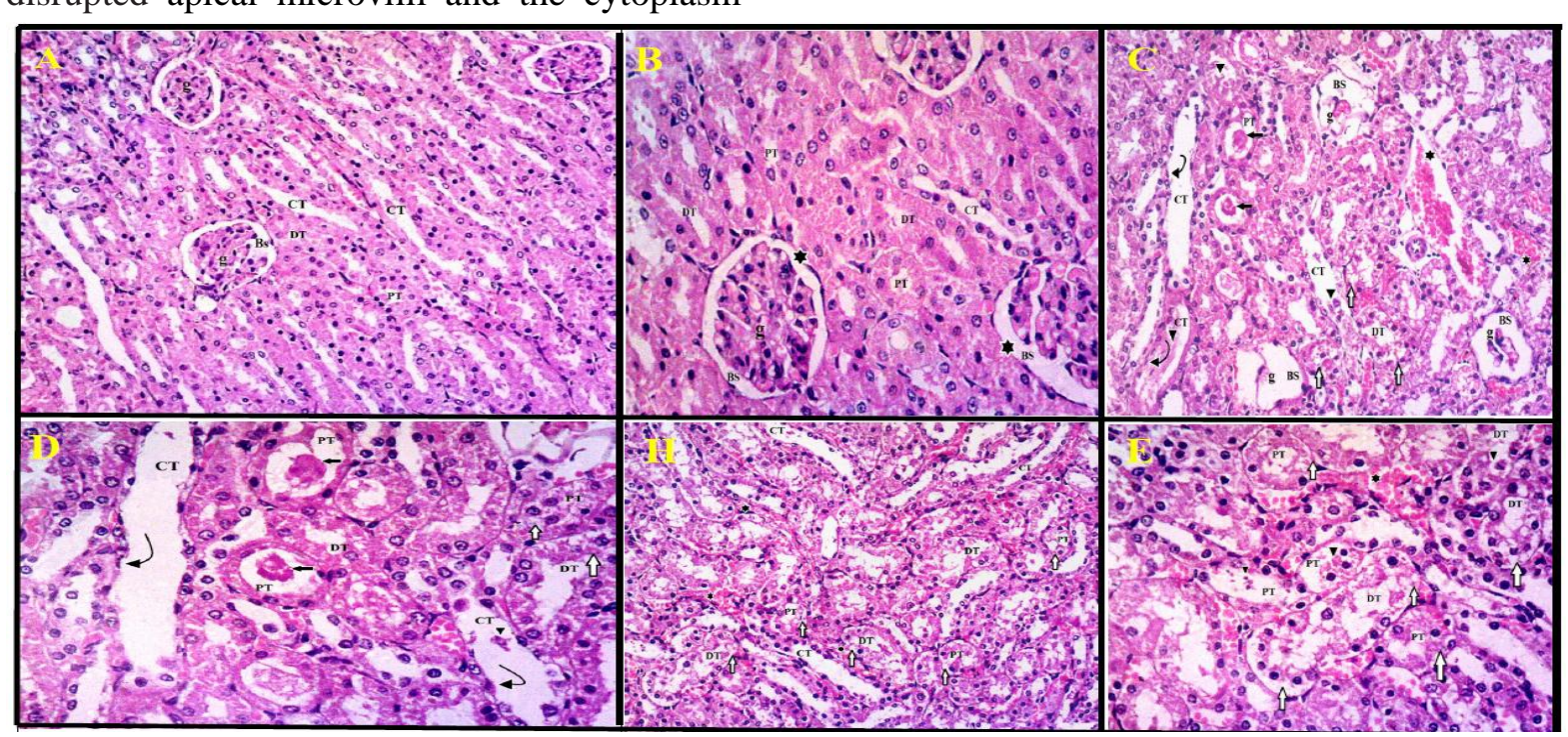

Plate 5 :( A, B): photomicrograph of the kidney section of pregnant female albino rat of the control group showing normal renal corpuscles consisting of glomeruli (g) and Bowman's space (BS). Narrow proximal convoluted tubules, wider distal convoluted tubules (DT) and elongated collecting tubules (CT), (B): normal glomeruli (g), Bowman's space (BS) surrounded by parietal layer of Bowman's capsule (star * ). Notice, normal cubical epithelial lining of narrow proximal convoluted tubules, wider distal convoluted tubules (DT) and elongated collecting tubules (CT) (H\&E X 200,400). (C, D, H and E): (C): photomicrograph of the kidney from pregnant female rat treated with $10 \mathrm{~nm}$ AuNSs showing congested renal and peritubular capillaries (star*), shrunken and lobulated glomeruli (g) with collapsed tuft and widening of the Bowman's space (BS). The tubular epithelium of both proximal (PT) and distal (DT) convoluted tubules showing cloudy swelling, (D): vacuolated cytoplasm (white arrow仓) and darkly stained nuclei. Notice, hyaline casts ( - ) in their lumen. Mildly dilated collecting tubules (CT) with flat dark nuclei of the lining epithelium (curved arrow). Notice, sloughing of some epithelial cells (arrow head ) capillaries (star*). Cloudy swelling, vacuolated cytoplasm (white arrow 0 ) and darkly stained nuclei in the tubular epithelium of both proximal (PT) and distal (DT) convoluted tubules. Mildly dilated collecting tubules (CT) with dark nuclei of the lining epithelium, $(\mathbf{E})$ : vacuolated cytoplasm (white arrow $\hat{\text { ) }}$ and darkly stained apoptotic nuclei in the tubular epithelium of proximal (PT) and distal (DT) convoluted tubules. Notice, sloughing of some epithelial cells (arrow head $\mathbf{\nabla}$ ) inside the lumen of some tubules and congested peritubular capillaries (star*).

(H\&E X 200, 400, 200 and 400). 


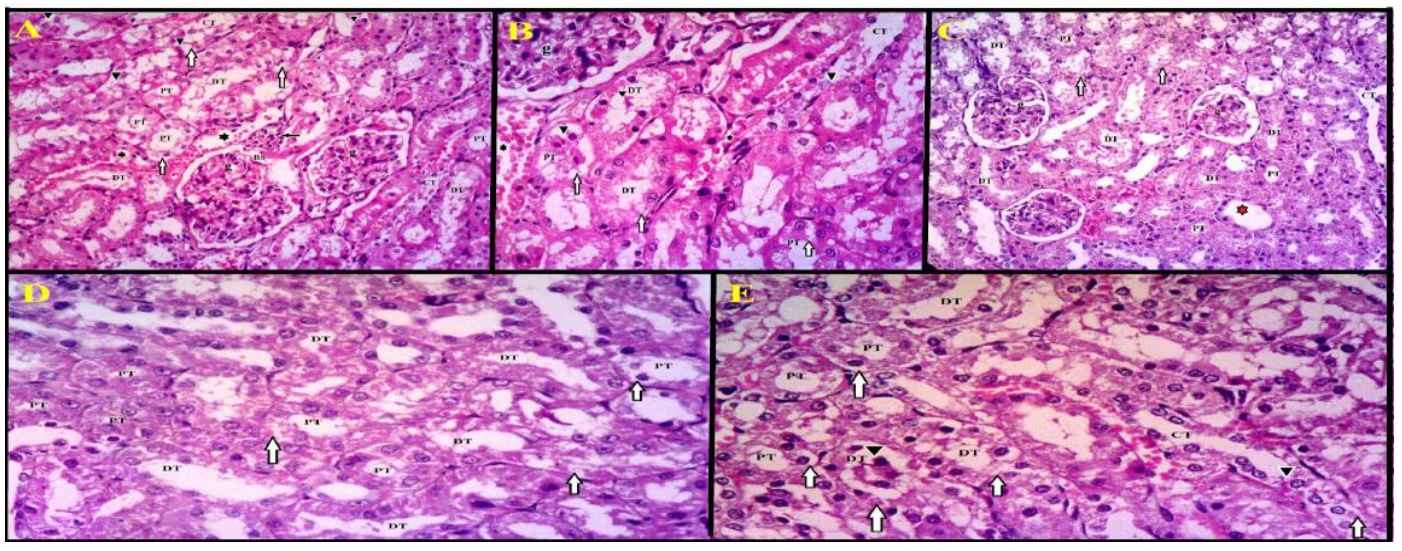

Plate 6: (A, B): Light micrograph in the renal section of $25 \mathrm{~nm}$ AuNSs-treated rat showing glomerular congestion $(\mathrm{g})$ with capillary dilatation and congested peritubular capillaries (star*). Cloudy swelling, vacuolated cytoplasm (white arrow $仑$ ) and darkly stained nuclei in the tubular epithelium of both proximal (PT) and distal (DT) convoluted tubules. Notice, sloughing of some epithelial cells (arrow head $\mathbf{\nabla}$ ) inside the lumen of some dilated collecting tubules (CT) .Mononuclear cellular infiltration (†) can also be observed, (B): demonstrating glomerular congestion $(\mathrm{g})$ and congested peritubular capillaries (star*). Cloudy swelling, vacuolated cytoplasm (white arrow $\hat{\text { ) }}$ ) and darkly stained nuclei in the tubular epithelium of both proximal (PT) and distal (DT) convoluted tubules and sloughing of some epithelial cells (arrow head $\boldsymbol{\nabla}$ ) inside their lumen and inside some dilated collecting tubules (CT) can also be observed (H\&E X 200, 400). (C, D and E): Light micrograph from kidney section of $50 \mathrm{~nm}$ AuNSs-treated rat showing preservation of normal renal glomeruli and tubules. Glomerular congestion $(\mathrm{g})$ and cloudy swelling, vacuolated cytoplasm (white arrow $\hat{\text { ) }) ~ i n ~ t h e ~ t u b u l a r ~ e p i t h e l i u m ~ o f ~ s o m e ~ p r o x i m a l ~(P T) ~ a n d ~ d i s t a l ~(D T) ~ c o n v o l u t e d ~ t u b u l e s . ~}$ Notice, empty space lined by squamous cells (red star). (D): showing normal narrow proximal convoluted tubules (PT), wider distal convoluted tubules (DT) lined by normal cubical epithelium. Notice, cloudy swelling, vacuolated cytoplasm (white arrow $\hat{\text { s) }}$

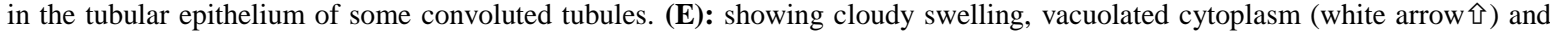
darkly stained nuclei in the tubular epithelium of both proximal (PT), distal (DT) convoluted tubules and collecting tubules (CT). Sloughing of some epithelial cells (arrow head $\mathbf{v}$ ) inside their lumen can also be observed (H\&E X 200, 400 and 400).

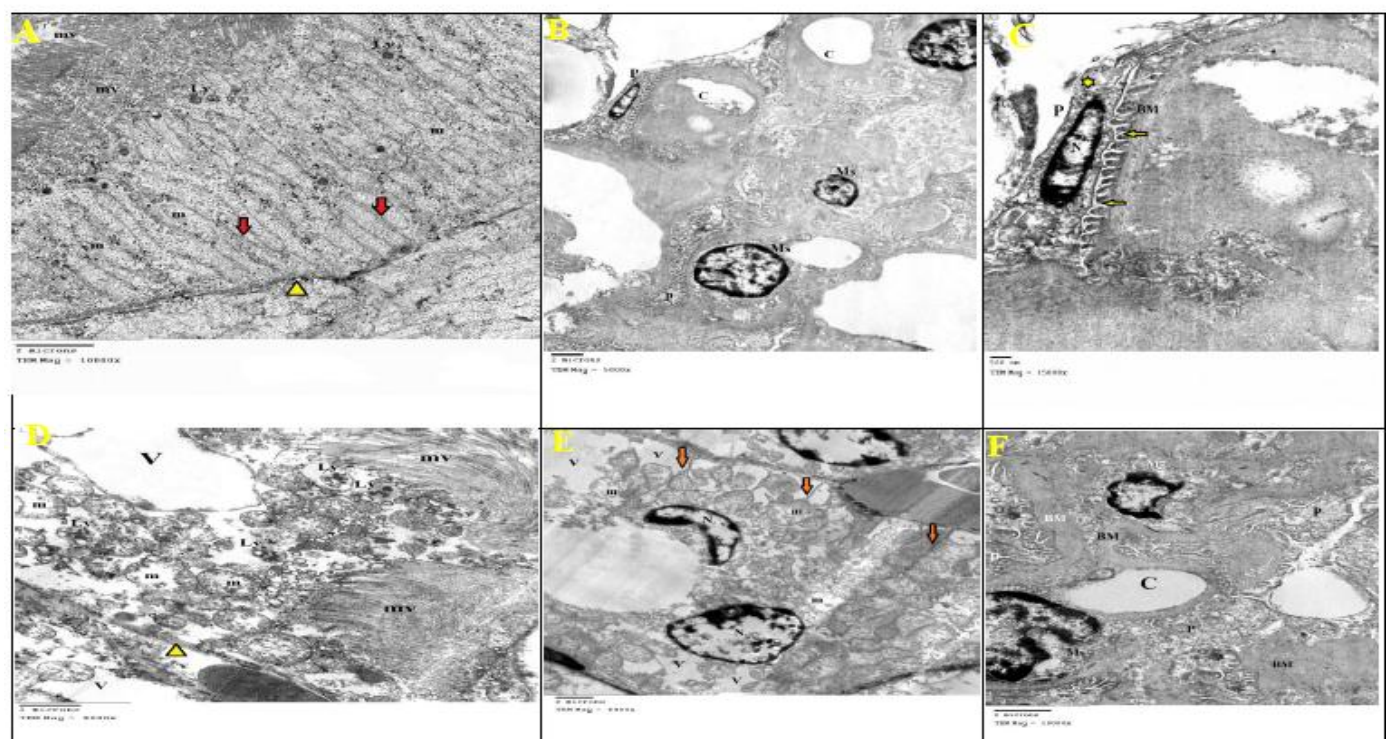

(Plate 7 A, B and C): Transmission electron micrograph of the proximal convoluted tubule revealed normal tubular basement membrane $(\Delta)$ with basal infoldings $(\sqrt{ })$. Cytoplasm contained many elongated mitochondria $(\mathrm{m})$ and a few electron dense lysosomes (Ly). Notice, the characteristic luminal long densely packed microvilli (mv). (B): showing glomerular tissue from the control group showing a multiple glomerular capillary $(\mathrm{C})$, podocytes with primary and secondary processes $(\mathrm{P})$ and mesangeal cells $(\mathrm{Ms})$. (C): showing a podocyte $(\mathrm{P})$ with central nucleus $(\mathrm{N})$ with characteristic well-formed primary (wyellow star) and secondary foot processes ( $<$ yellow arrows) with regular spacing between them resting on the glomerular basement membrane (BM) (Magnification X 10000,5000 and 15000). (D, E and F): An electron micrograph of the kidney section of $10 \mathrm{~nm}$ AuNSstreated rat displaying a part of the proximal convoluted tubule with its characteristic luminal microvilli (mv). There are numerous vesicles, hydropic degeneration and vacuolation of the cytoplasm (V). Notice, degenerated mitochondria with disrupted cristae $(\mathrm{m})$, electron-dense lysosomes (Ly) varying in size and shape and thickening of the tubular basement membrane ( $\Delta$ yellow arrow head). (E): Showing a part of the distal convoluted tubule with basal infoldings ( $\checkmark$ red arrow) apparent in some areas. The tubular cells revealed hydropic degeneration, vacuolation $(\mathrm{V})$ of the cytoplasm. Note shrunken pyknotic nuclei $(\mathrm{N})$ with irregular nuclear envelope, margination of heterochromatin is seen as well. Notice also, degenerated mitochondria with disrupted cristae (m). (F): displayed multiple glomerular capillaries $(\mathrm{C})$, podocytes with primary and secondary processes $(\mathrm{P})$ and mesangeal cells $(\mathrm{Ms})$ with electron-dense cytoplasm. Notice, apparent thickening of basement membrane (BM) in certain areas (Magnification X 8000, 6000 and 10000). 


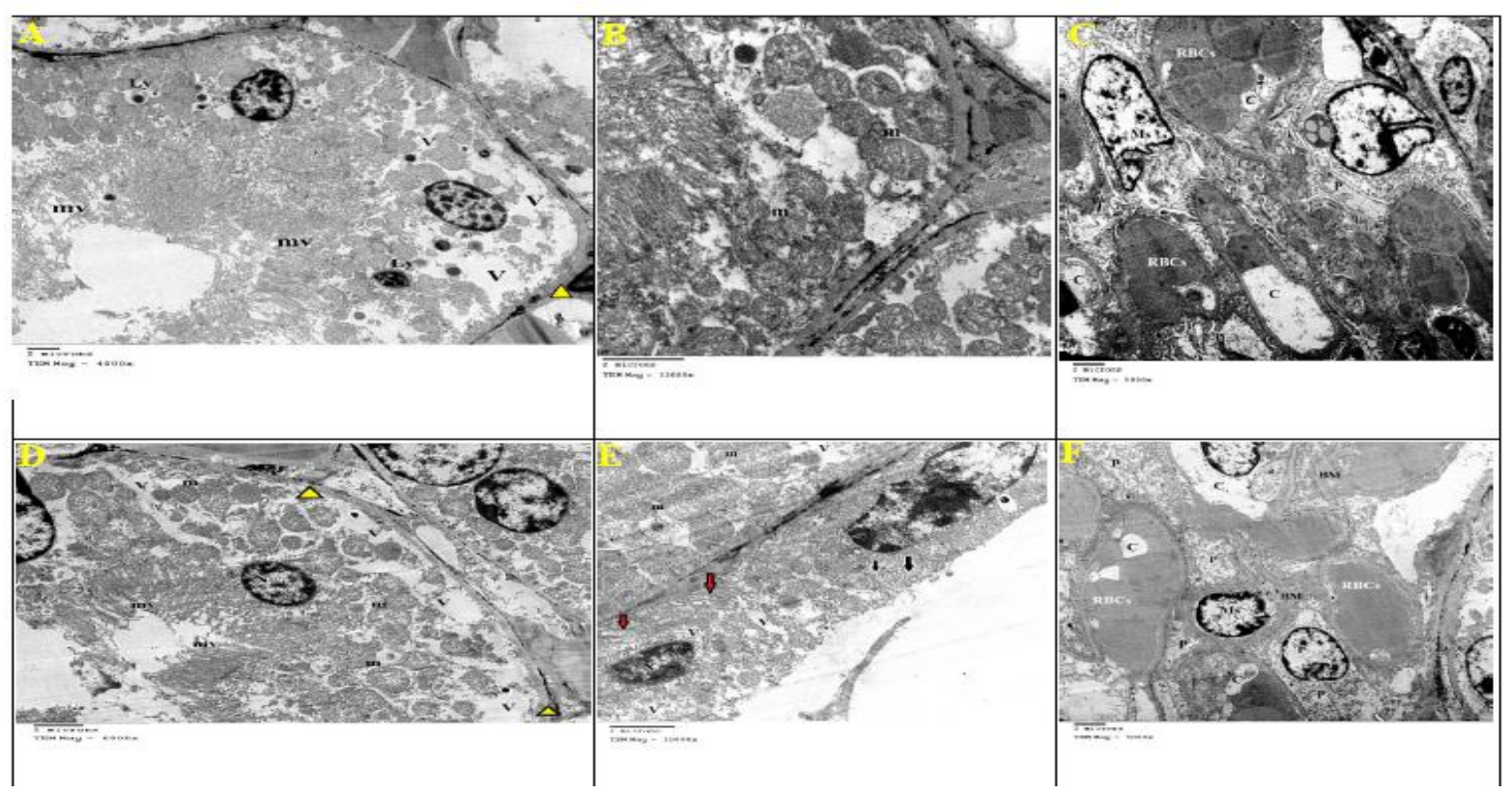

Plate 8 :( A, B and C): An electron micrograph of the kidney section of $25 \mathrm{~nm}$ AuNSs-treated rat displaying part of the proximal convoluted tubule with its characteristic microvilli (mv). Hydropic degeneration, vacuolation of the cytoplasm (V) and electron-dense lysosomes (Ly) of variable size and shape are seen. Note

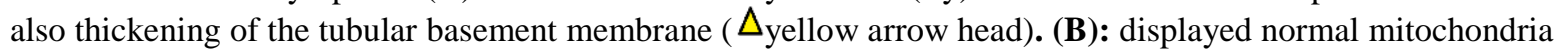
(m) with intact cristae and electron dense internal matrices. (C): Showing multiple congested glomerular capillaries (C) containing red blood corpuscles (RBCs), podocytes (P) and mesangeal cells (Ms) Notice, apparent thickening of basement membrane (BM) in certain areas (Magnification X 4000, 12000 and 5000). (D, E and F): An electron micrograph of the kidney section of $50 \mathrm{~nm}$ AuNSs-treated rat displaying part of the proximal convoluted tubule with its characteristic microvilli (mv). Hydropic degeneration, vacuolation of the cytoplasm (V) and degenerated swollen mitochondria $(\mathrm{m})$. Notice, shrunken pyknotic nuclei $(\mathrm{N})$ with margination of heterochromatin is seen as well. Thickening of the tubular basement membrane ( $\Delta_{\text {yellow }}$ arrow head). (E): Showing a part of the distal convoluted tubule with basal infoldings ( red arrow) are apparent in some areas. The tubular cells revealed numerous vesicles (black arrow), vacuolation (V) of the cytoplasm. Note shrunken pyknotic nuclei $(\mathrm{N})$ with irregular nuclear envelope, margination of heterochromatin is seen as well. Notice also, swollen mitochondria with intact cristae (m). (F): displaying multiple congested glomerular capillaries (C) containing red blood corpuscles (RBCs), podocytes (P) and mesangeal cells (Ms). Notice, apparent thickening of basement membrane (BM) (Magnification X 6000, 10000 and 5000).

\section{DISCUSSION:}

Hypotheses which have been proposed about probable damages resulting from nanotechnology are threatening the development of nanotechnology, unless the correct information about what the risks are and how to avoid it (Zhang et al., 2010). Investigations in the present study emphasized on those results of researches and showed low to high abnormalities in liver and kidney, as in pregnant rats treated with $10 \mathrm{~nm}$ showed in liver section dilated and congested hepatic blood vessels, widespread vacuolated and ballooned hepatocytes with early apoptotic changes, the Kupffer cells were hypertrophied within the dilated blood sinusoids in some areas Also pregnant treated with $25 \mathrm{~nm}$ and 50 AuNSs showed preserving the normal hepatic architecture. However, degenerative and apoptotic changes were encountered in a few hepatocytes this agreement with Abdelhalim and Jarrar, (2011) when reported that in comparison with the control rats, the exposure to AuNSs doses has produced inflammatory cell infiltration, Kupffer cells hyperplasia, central veins intima disruption, hepatic strands dilatation and occasional fatty change together with a loss of normal architecture of the hepatic strands and they decided that the exposure to intraperitoneal administration of $10 \mathrm{~nm}$ AuNSs 
produced histological alterations in the different rat organs: In the liver: the hepatocytes, portal triads and the sinusoids, which were mainly vacuolar with hydropic degeneration, cytoplasmic hyaline vacuolation, polymorphism, binucleation. (Park \& Bae, 2010 and Abdelhalim, 2011) Nanoparticles released into the blood have been shown to accumulate, with toxic effects in the liver, kidney, and heart, causing scattered cytoplasmic vacuolization, appearance of chronic inflammatory cells, and congested and dilated blood vessels. The histological alterations induced in liver and kidney organs of the rats by administration of AuNSs where size dependent were smaller ones induced more effects and were related with time exposure of AuNSs. These alterations related to AuNSs toxicity became unable to deal with the accumulated residues resulting from the metabolic and structural disturbances caused by these AuNSs, the histological alterations suggested that AuNSs might interact with proteins and enzymes of the different organs rats. (Abdelhalim and Jarrar, 2011). For the kidney in this study the pregnant rat treated with $10 \mathrm{~nm}$ AuNSs showed glomerular, tubular and interstitial histological alterations, congested renal and peritubular capillaries, Shrunken renal corpuscles with collapsed tuft and wide Bowman's space. For rats treated with $25 \mathrm{~nm}$ AuNSs. The congested glomeruli showed capillary dilatation. Renal tubules appeared dilated and showed degenerative changes mostly cloudy swelling, vacuolated cytoplasm and pyknotic nuclei of the lining tubular epithelium this was in agreement with Wang et al.( 2007) when reported that the rats treated with AuNSs showed cloudy swelling, vacuolar degeneration, hyaline droplets and casts. The glomeruli showed moderate congestion with no hypercelluraity, they believed that nanoparticles have been stored in the cells of kidney and caused the pathological changes and nephron-like toxicity in the form of inflammation of the glomeruli of the kidney

(De Jong et al., 2008). The smaller AuNSs of 5-15 $\mathrm{nm}$ have wider organ distribution than that of large AuNSs of 50-100 nm It has been found that AuNSs with a long blood circulation time can accumulate in the liver and kidney, and significantly affect the gene expression (Cho et al., 2009 and Vandebriel \& De Jong, 2012). Nanoparticles may induce the formation of highly reactive oxygen species, including hydrogen peroxide, hydroxyl radicals, and superoxide anions, all of which can cause oxidative damage to animal cells.

TEM findings in the current study revealed numerous important ultrastructure changes in liver cells and kidney for pregnant rats treated with $10 \mathrm{~nm}$ AuNSs where marked cytopathological changes were seen in hepatocyte, dramatic pyknotic hyperchromatic irregular nucleus with deteriorated chromatin condensation and the cytoplasm was highly vacuolated with rarified and abnormal mitochondria. This agreement with (El-Daly, 2017) where rats treated with nanomaterial showed vacuolated cytoplasm, damaged mitochoderia and rER and pale nucleus with irregular contour. (Ansari et al., 2016) according to rats were treated with AgNPs were watched irregularities in nuclear membrane, chromatin condensation, degenerated hepatocytes with shrunken and atrophic nucleus, vacuolated cytoplasm, pleomorphic and swollen mitochondria with distorted cristae, irregularities in rough endoplasmic reticulum with extensive dilation. These observations are suggestive of early necrosis and apoptosis in hepatocytes. Ultrastructural observations in the present study for the kidney rats treated with different size of AuNSs,evidenced the cytoplasm nearly devoid of organelles except from many pinocytotic vesicles, swollen mitochondria, swelling of primary foot processes and fusion of the secondary foot processes of the podocyte in certain areas and long densely packed microvilli at the luminal surface of the proximal tubular cells. This is in agreement with Ansari et al.,( 2016) where treated rats with AuNSs showed change in mitochondria particularly evident in proximal convoluted tubules, a significant decrease in length of 
filamentous mitochondria was also noticed, Hypertrophied and fused podocytes and chromatin condensation, dilation of rough endoplasmic reticulum, cytoplasmic vacuoles and thickened glomerular basement membrane were also detected that may led to the necrosis and apoptosis of the cells. Chen et al., (2009) Concluded that accumulation of nanoparticles was observed in the cytoplasmic matrix and organelles including mitochondrial matrix as electron-dense material and dilated rER in a dose dependent manner, the particles in Kupffer cell were detected. Moreover, these accumulations were more obvious in perinuclear membrane and inter nuclear matrix, may be due to the smaller particles sizes that are easier to enter the cells than larger size so the particle deposition in liver may lead to hepatic lesions. However, the physical and chemical properties of nanomaterial are expected to cause significant effects on the behavior and properties of macromolecules, cells and body parts. Saman et al.( 2013) using $\mathrm{Ag}$ NPs, noted that the particles were concentrated within membrane-bound vesicles point to either effective removal from the cytoplasm after diffusion through the membrane and incorporation into phagosomes or lysosomes, or uptake by mechanisms involving membrane incorporation of particles. Long et al.,( 2006) reported that the presence of TiO2-NPs in these manners could facilitate generation and accumulation of ROS and oxidative stress that may be the main cause of the ultrastructure changes in the hepatocytes as swelling, perforations and disintegration of mitochondria, rER, irregularity in nuclear envelope and condensed as well as fragmented chromatin, the developed oxidative stress increases lipid peroxidation of membranes of mitochondria and rER permeability leading to disturbance in ATP and the intracellular calcium ions levels.

\section{CONCLUSION:}

The results of the present work indicated that all sizes of Nano gold can cause side effect on pregnant rats but 10 AuNSs caused side effect more than 25, 50 AuNSs.

\section{REFERENCES:}

1- Abdelhalim, M. A. (2011): Gold nanoparticles administration induces disarray of heart muscle; hemorrhagic, chronic inflammatory cells infiltrated by small lymphocytes, cytoplasmic vacuolization and congested blood vessels. Lipids Health Dis, 10:233-241.

2- Abdelhalim, M. A. K. and Jarrar, B. M (2011): The appearance of renal cells cytoplasmic degeneration and nuclear destruction might be an indication of AuNSs toxicity. Lipids Health Dis, 10: 147.

3- Ansari, M. A.; Shukla, A.K.; Oves, M. and Khan, H. M. (2016): Electron microscopic ultrastructural study on the toxicological effects of AAuNSs on the liver, kidney and spleen tissues of albino mice. Enviro. Toxico. Pharma, (16): 1382-6689.

4- Chen, J.; Dong, X.; Zhao, J. and Tang, G. (2009): In vivo acute toxicity of titanium dioxide nanoparticles to mice after intraperitioneal injection. J Appl Toxicol, 29: 330-337.

5- Cho, W. S.; Cho, M.; Jeong, J.; Choi, M.; Cho, H. Y.; Han, B. S.; Kim, S. H.; Kim, H. O.; Lim, Y. T. and Chung, B. H. (2009): Acute toxicity and pharmacokinetics of $13 \mathrm{~nm}$ sized PEG-coated AuNSs. Toxicol. Appl. Pharmacol, 236(1): 16-24.

6- Connor, E.E.; Mwamuka, J.; Gole, A.; Murphy, C.J. and Wyatt, M.D.(2005): AuNSs are taken up by human cells but do not cause acute cytotoxicity. Small, 1: 325-327.

7- Davenport, H. A. (1960): Histological and histochemical techniques. Saunders, W.B. Co., Philadelphia and London: 212-285.

8- De Jong, W. H.; Hagens, W.I.; Krystek, P.; Burger, M. C.; Sips, A. J. A. M. and Geertsma, R. E. (2008): Particle sizede pendent organ distribution of AuNSs after intravenous administration. Biomaterials, 29:1912-1919.

9- El-Daly, A. A. (2017): The histopathological, ultrastructural and immunohistochemical effects of intraperitoneal injection with titanium dioxide nanoparticles and titanium dioxide bulk on the liver of the albino Mice. J Anim. Health. Behav. Sci, 1:1.

10- Gunawan, C.; Teoh, W.Y.; Marquis, C.P. and Amal, R. (2011): Cytotoxic origin of copper(II) oxide nanoparticles: comparative studies with micron-sized particles, leachate and metal salts. ACS Nano, 5(9):7214-7225.

11- Jia, H.Y.; Liu, Y.; Zhang, X.J.; Han, L.; Du, L. B.; Tian, Q. and Xu, Y.C. (2009): Potential 
oxidative stress of AuNSs by induced-NO releasing in serum. J. Am. Chem. Soc, 131: 4041

12- Johannessen, J. V. (1978): Electron microscopy in human medicine. McGraw-Hill International Book Co., New York

13- Long, T.C.; Saleh, N.; Tilton, R. D.; Lowry, G. V. and Veronesi, B. (2006): Titanium dioxide (P25) produces reactive oxygen species in immortalized brain microglia (BV2): implications for nanoparticle neurotoxicity. Environ. Sci. Technol, 40: 4346-4352.

14- Pan, Y.; Neuss, S.; Leifert, A.; Fischler, M.; Wen, F.; Simon, U.; Schmid, G.; Brandau, W. and Jahnen-Dechent, W.( 2007): Sizedependent cytotoxicity of gold nanoparticles. Small 3, 1941-1949.

15- Park, E. and Bae, E. (2010): Repeated-dose toxicity and inflammatory responses in mice by oral administration of silver nanoparticles. Environ Toxicol Pharm, 30:162-168.

16- Saman, S.; Moradhaseli, S.; Shokouhian, A. and Ghorbani, M. (2013): Histopathological effects of $\mathrm{ZnO}$ nanoparticles on liver and heart tissues in Wistar rats. Adv Biores, 4: 83-88.

17- Studer, A. M.; Limbach, L. K.; Van Duc, L.; Krumeich, F.; Athanassiou, E. K. and Gerber, L. C. (2010): Nanoparticle cytotoxicity depends on intracellular solubility:comparison of stabilized copper metal and degradable copper oxide nanoparticles. Toxicol Lett, 197(3):169-174.

18- Takahashi, H.; Niidome, Y.; Niidome, T.; Kaneko, K.; Kawasaki, H. and Yamada, S. (2006): Modification of gold nanorods using phosphatidylcholineto reduce cytotoxicity. Langmuir, 22 (1): 2-5.

19- Tedesco, S.; Doyle, H.; Blasco, J.; Redmond, G. and Sheehan, D. (2010): Oxidative stress and toxicity of AuNSs inMytilusedulis. Aquat. Toxicol, 100: 178-186.

20- Turkevich, J.; Stevenson, P.C. and Hillier, J. (1951): A study of the nucleation and growth processes in the synthesis of colloidal gold, Discussions of the Faraday Society, 11: 55-75.

21- Vandebriel, R. J. and De Jong, W. H. (2012): A review of mammalian toxicity of $\mathrm{ZnO}$ nanoparticles. Nanotechnol. Sci .Appl, 5:61-71.

22- Wang, J.; Zhou, G.; Chen, C. and Yu, H. (2007): Acue Toxicity and Biodistribution of Different Sized Titanium Dioxide Particles in Mice after Oral Administration. Eur. J. Toxicol. Hyg. Environ, 168: 176-185.

23- Zhang, X. D.; Wu, H.Y.; Wu, D. and Wang, Y.Y. (2010): Toxicologic Effects of Gold Nanoparticles In vivo by Different Administration Routes. Int $\mathrm{J}$ nanomedicine, 5: 771-781.

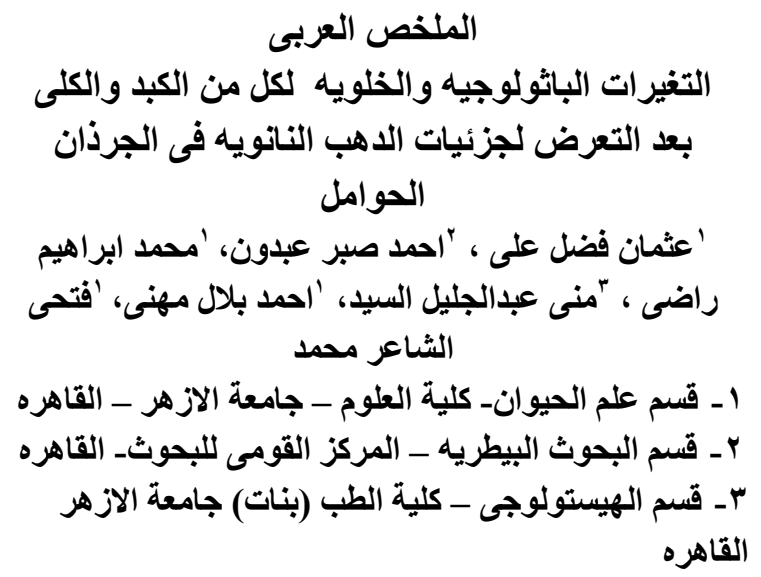

